\title{
LANGUES, SAVOIRS ET POUVOIRS EN MILIEU MAURE
}

\author{
Catherine Taine-Cheikh
}

Dans la Mauritanie contemporaine, la revendication de l'arabe fait figure de principe fondamental de l'entité maure. Pourtant les populations sahariennes du Sahara occidental furent longtemps berbérophones et mirent sans doute plusieurs siècles à changer de « langue maternelle ». C'est d'abord sur cette arabisation dialectale que je m'interrogerai, en montrant qu'on ne peut pas la couper complètement du mouvement d'islamisation qui l'a précédée et qui semble avoir influé fortement sur l'image que ces populations pouvaient se faire d'elles-mêmes.

$\mathrm{Si}$, à partir du $18^{\mathrm{e}}$ siècle, l'arabité culturelle de cette région prend des contours plus nets, il faut prêter attention au fait que, derrière l'unité apparente du terme " arabe», se cache une dualité mouvante - ce qu'on appelle la diglossie arabe littéraire / dialecte - , à laquelle correspondent deux formes culturelles distinctes. Je propose ici de caractériser la culture savante des zwâye comme «aurale » (par delà la dichotomie écrit / oral) ${ }^{1}$ par opposition à l'oralité de la culture populaire en dialecte, telle qu'elle s'incarne notamment chez les musiciens-chanteurs.

Dans la dernière partie, j'étudierai l'évolution récente de la diglossie et les tentatives faites pour échapper aux difficultés posées par cette opposition, qu'elles restent à l'intérieur du domaine arabe ou qu'elles choisissent d'en sortir. Nous verrons que là encore le poids de l'idéologie se fait sentir et que les représentations des locuteurs influent sur leurs productions langagières ${ }^{2}$.

1. C'est à J.-L. Siran et à son compte-rendu de De la voix au texte. L'ethnologie contemporaine entre l'oral et l'écrit (1998) que je dois l'idée d'une opposition significative entre «aural » et « oral ». Partant notamment des articles de L. Mabru « De l'écriture du corps dans les pratiques musicales à transmission «orale »: le cas du fifre en Bazadais » et de N. Belmont «L'Ecriture des contes », il suggère une opposition entre l'oralité des contes «comme Autre de l'écrit» et l'auralité de la musique, inséparable d'une certaine écriture (due à l'"inscription corporelle » par « voie sensorielle, 'aurale' et visuelle »).

2. Je fais ici plusieurs propositions et suis donc très reconnaissante envers tous ceux qui ont bien voulu lire les versions provisoires de cet article. Je remercie en particulier A. W. O. Cheikh et A. Roth pour leurs remarques et suggestions, qu'elles aient pu être intégrées dans la version définitive ou qu'elles soient mises en attente pour des développements ultérieurs. 


\section{La langue du père}

Les deux grandes sociétés nomades de l'Afrique de l'Ouest - la maure et la touarègue - semblent présenter, dans leurs structures profondes comme dans leurs manifestations de détail, d'énormes ressemblances. Pourtant, alors que les Maures sont considérés par les Touaregs comme de proches parents qui ont perdu les vraies valeurs (H. Claudot-Hawad, 1996), les seconds sont pour les premiers des a $a^{j} \hat{a} m$ c'est-à-dire, au sens littéral, des « étrangers » car ils ne parlent pas arabe. C'est ce paradoxe que je tenterai d'abord d'éclairer et qui est lié, bien sûr, au recul du berbère dans une partie du Sahara occidental.

\section{Les Zénagas en question}

Dans les premiers siècles de notre ère et, au moins, jusqu'aux premiers mouvements d'islamisation, on n'a pas de raison de supposer que les diverses communautés berbérophones de l'Afrique de l'Ouest se présentaient sous la forme de grandes entités différenciées, à l'instar de ce que l'on aurait tendance à nommer, par projection à partir du présent, le monde chleuh, le monde touareg et le monde zénaga ${ }^{3}$.

Quels que soient, cependant, les rapports anciens et continus avec les populations sédentaires du Sud-marocain - cette région ayant correspondu, semble-t-il, à une zone de transition bien avant l'arrivée des Arabes -, ce que l'on peut connaître du monde zénaga de l'époque est à chercher dans les manuscrits arabes sous l'appellation șanhāja et apparaît étroitement lié à l'élevage chamelier. De ce point de vue, il apparaît que la vie saharienne, rendue possible grâce à l'introduction du dromadaire domestique, rapproche beaucoup plus le monde zénaga du monde touareg que du monde chleuh et que ce n'est pas un hasard si le terme de al-mulattamūn («les [hommes] voilés ») a pu s'appliquer aux habitants du désert, Zénagas comme Touaregs, mais n'a jamais concerné les Chleuhs ${ }^{4}$.

Même si le Sahara a fait très tôt l'objet d'une certaine répartition spatiale entre les différents groupes de Berbères nomades (P. Bonte, 1998: 43), c'est peut-être seulement avec la pénétration de l'Islam que l'on doit situer les véritables prémisses de la séparation entre Zénagas et Touaregs, les premiers n'émergeant comme entité que par le mouvement même qui va les conduire à se

3. Les résultats actuels de mes enquêtes linguistiques sur le zénaga m'inclinent en effet à poser un bilan comparatif extrêmement nuancé. Derrière les particularités de ce dialecte explicables par la situation en complète autarcie, apparaît une certaine parenté avec les dialectes touaregs proximité à la fois d'évolution (commune parfois avec des dialectes plus orientaux, également considérés comme périphériques) et par rapport au fond lexical - mais l'intercompréhension actuelle (là encore à cause, sans doute, de l'importance du vocabulaire commun) semble plus grande avec les voisins chleuhs du Nord qu'avec les proches voisins touaregs de l'Est (l'avenir dira s'il faut nuancer cette affirmation par rapport aux voisins touaregs plus lointains de l'Aïr). Cf. C. Taine-Cheikh, 1999.

4. «Cette seconde classe des Sanhâja, les Mulaththamûn, habite les déserts au delà des sables du Sahara, vers le Sud. Ils ont parcouru en profondeur cette région plusieurs siècles avant la conquête islamique, depuis un temps immémorial. » (Ibn Khaldûn, in Cuoq, 1974 : 332). 
dissoudre dans une autre, celle des Maures. C'est en partie l'hypothèse défendue par A.W. O. Cheikh ${ }^{5}$ et cela coïncide assez bien avec la représentation des Touaregs, qui voient dans leurs voisins Maures des « Berbères islamisés » 6 .

Historiquement, les débuts de l'Islam en Afrique de l'Ouest ne sont connus que bien imparfaitement mais, pendant longtemps, la langue berbère a dû jouer un rôle primordial dans la vie religieuse et il est probable que la diversité (fonctionnelle et linguistique) qu'on peut observer de nos jours tient au fait qu'elle n'a pas résisté partout de la même manière à la pression de l'arabe. Sans doute peut-on voir une trace de cette résistance au fait que seuls certains dialectes - tous très méridionaux puisqu'il s'agit, outre la tamašaq et le zénaga, de la tašlhit et des parlers algériens de Ouargla et du Mzâb ${ }^{7}$ - ont conservé des termes typiquement berbères pour nommer les cinq prières islamiques. En ce qui concerne l'Afrique de l'Ouest, ce fait peut être mis en rapport avec d'autres indices tels que l'existence de manuscrits en berbère à thème religieux, écrits en caractères $\operatorname{arabes}^{8}$, ou l'habitude de commenter le Coran et les textes fondamentaux de l'Islam en berbère ${ }^{9}$.

Dans l'histoire des rapports entre le berbère et l'islam, il est probable que certains changements ont pesé lourd pour le devenir du berbère, tels ceux qui s'opérèrent au Maroc avec le pouvoir mérinide et qui virent notamment la fin du prêche et de l'appel à la prière en berbère (A. Bounfour, 1994a : 36 et sq.).

Si la place du berbère était encore bien assurée à l'époque du pouvoir almohade (dont on connaît le lien avec la région du Sud-marocain), on peut supposer qu'il en allait de même à l'époque antérieure des Almoravides. De ce point de vue, il ne faut sans doute pas se laisser abuser par le fait que le nom de ce mouvement est une forme hispanisée du terme arabe murābițūn, lui-même dérivé du nom ribāt, lieu tout à la fois de garnison et de prière (G. Camps, 1983 : 13). À défaut de renseignements précis sur la connaissance qu'on avait, au $11^{\mathrm{e}}$

5. A propos de la lettre d'un certain Al-Lamtūnī datée du $15^{\mathrm{e}}$ siècle, A. W. O. Cheikh souligne tous les éléments communs aux deux sociétés sanhajiennes de l'époque et montre que la description s'applique peut-être mieux encore à la situation du Sahara occidental qu'à celle du Sahara central (1985 : 163 et sq.)

6. Par opposition aux Maures, les Touaregs — du moins les nobles d'entre eux - semblent avoir opposé une grande résistance à l'islam : «Les récits historiques touaregs insistent sur le caractère étranger de l'islam et son importation relativement récente par les Arabes. La religion est considérée comme un engagement personnel qui ne concerne que l'individu et n'intervient pas dans la définition de l'identité touarègue. » (H. Claudot-Hawad, 1993 : 154).

7. Voici les noms des cinq prières en zénaga (la spirantisation n'y est pas notée) : tnezzett (litt. « matin »), tajbaran (« celle qui est plus rapide, qui devance »), tekkuzəon (《les 4èmes»), tnuty̌šn (« celles de la tombée de la nuit») et tiñudaššsn («celles du sommeil »). Pour les autres parlers et quelques explications complémentaires, cf. K. Naït-Zerrad, $1998: 62-4$.

8. Y. O. Elbara a découvert et étudié un manuscrit de ce type en Mauritanie, dont l'ancienneté pourrait rivaliser avec le célèbre L'océan des pleurs du lettré sud-marocain M'hemd ou Ali Awzal (cf. H. Jouad, 1987).

9. C'est ainsi que notre informateur zénagophone nous dit avoir fait ses études voici seulement vingt ou trente ans, dans une mahađ̛̣a du Sud-ouest mauritanien. On pourra faire le rapprochement avec les pratiques traditionnelles des Ineslimen, chez les Touaregs de l'Ouest, mais on notera que dans ce cas, comme chez les Chleuhs, il s'agit peut-être surtout d'enseignement islamique de base (A. Bounfour, 1994a : 36) alors que chez notre informateur, il s'agissait d'études supérieures. 
siècle, de l'arabe coranique, on peut supposer toutefois que les difficultés de langue n'étaient pas forcément étrangères au besoin que ressentit Brahim Ibn Yahya al-Gdâli, après un pélerinage à la Mecque, de faire appel à un prédicateur étranger ('Abd Allâh Ibn Yasîn de la tribu sud-marocaine des Jazûla, formé à Grenade), pour l'aider à réformer la religion des gens de chez lui.

Avec le mouvement almoravide, - épisode marquant de l'histoire de l'Afrique de l'Ouest —, se constitue sans doute une première différenciation importante entre le monde zénaga et le monde touareg.

\section{La mère bâillonnée}

Dans le cas de l'émergence de la société maure, on peut dire que d'une certaine manière - mythiquement ou, du moins, métaphoriquement - on est passé, au plan (socio)linguistique, de la langue de la mère à la langue du père, tout comme on est passé, d'une société cognatique, d'un principe de droit matrilinéaire à un principe de droit patrilinéaire $\left.{ }^{10}\right)$.

Si l'on reprend le mythe du Srîv Bū-bezzûl (litt. «le chérif au sein ») qui, $\mathrm{du}$ point de vue anthropologique, semble d'une grande richesse pour la compréhension de la société maure, on constatera qu'il s'interprète non seulement comme une arabisation généalogique, mais aussi comme une métaphore de l'arabisation linguistique.

Lorsque cette figure de la sainteté, présentée comme un personnage historique ayant vécu, selon les récits, au $15^{\mathrm{e}}$ ou $16^{\mathrm{e}}$ siècle, doit fuir son épouse berbère possédée par les diables et subvenir en plein désert aux besoins de son enfant - raison pour laquelle Dieu lui fait pousser un sein -, ce n'est pas uniquement du lait qu'il donnera à son fils, c'est aussi sa langue puisque le bébé est du même coup abstrait du bain langagier où il aurait dû grandir. Si, dans la société maure, les enfants de sexe masculin ont, vers sept ans, à quitter l'univers maternel et, plus généralement, féminin - ce qui a pu contribuer, en cas de cultures différentes, à affaiblir plus nettement la langue de la mère chez les garçons que chez les filles ${ }^{11}$ _, il s'agit là, sous la bannière ostensible, non seulement de l'Islam mais plus encore du Prophète lui-même (il s'agit, ne l'oublions pas, d'un shérif, donc d'un descendant du Prophète), de brûler toutes les étapes et de soustraire l'enfant à toute influence maternelle, c'est-à-dire berbère (et non musulmane). A ce niveau, la mère n'est pas empêchée de parler mais elle n'est plus entendue et ne peut que parler dans le vide. Le bâillonnement

10. Cf. P. Bonte, 1998 : 264 et sq. (« Rapprochées les unes des autres, les différentes versions que nous avons recueillies de ces récits centrés sur le Sharif Bûbazzûl, dessinent une configuration structurelle dont la dimension mythique est claire: par le biais d'une alliance matrimoniale du Sharif au sein d'une tribu berbère (ou parfois dans une population noire) est mise en scène l'affirmation simultanée de la patrilinéarité et de l'origine arabe, dans le contexte miraculeux du lait, de la nourriture qu'il fournit à son fils menacé », p. 265) et A. W. O. Cheikh, 1985 (notamment le chapitre III de la Ière partie, intitulé « Les Banī Hassān et 'le meurtre de la mère' »).

11. Par ailleurs, le fait que les tribus berbères aient été plutôt des donneurs de femmes (P. Bonte, 1998) a peut-être contribué à l'extinction du zénaga sous une forme très progressive car il est probable qu'en arrivant dans un campement arabophone, une femme berbérophone a des raisons limitées de transmettre sa langue. 
ne deviendra effectif qu'avec l'arabisation, quand la femme berbère perd son rôle social de véritable porte-parole.

Le rôle politique des femmes touarègues a été étudié notamment par $\mathrm{H}$. Claudot-Hawad (idem : 119 et sq.) mais le statut de porte-parole des femmes berbères est peut-être plus net encore au niveau symbolique. Dans le cérémonial de mariage kabyle tel que nous le décrit par exemple D. Abrous, la violence signe d'une compétition autour de l'honneur —, tient une grande place, mais cette violence est complètement dédoublée. «Pour cette citadelle à prendre d'assaut, la division des tâches est très clairement définie : aux hommes les armes, aux femmes la parole » (1992: 151), c'est-à-dire que les femmes rivalisent d'adresse verbale dans des joutes poétiques tandis que les hommes, de leur côté, affrontent l'épreuve du tir à la cible.

Ce sentiment de force de la parole féminine n'est peut-être pas sans rapport avec le tabou qui semble peser sur le nom même de «femme » et qui contribue à expliquer la remarquable variété des termes recueillis dans les divers dialectes (cf., entre autres, L. Galand, 1981 : 316 et sq.). En zénaga, le nom désignant la femme (tənəšt ${ }^{y} \partial m t$, pl. tənəššìmən) s'écarte radicalement de ce qu'on trouve ailleurs et semble devoir se comprendre comme «(la) musulmane » pour peu qu'on fasse le rapprochement avec la forme touarègue de aneslem pour « lettré musulman » (Fr. J.-M. Cortade, $1967: 320)^{12}$.

Tout ceci va dans le même sens que le mythe du Šrîv Bū-bezzûl : c'est au nom de la religion que la femme est muselée et que, symboliquement, la société berbère renonce à sa langue.

\section{Le langage des armes}

Si l'on rapproche le nom donné à la femme, du terme par lequel les zénagophones désignent les Maures (donc eux-mêmes pour peu qu'ils soient bilingues, car ils se reconnaissent comme locuteurs de zénaga mais non comme znâge ${ }^{13}$ ), on se rend compte que leur perception de l'entité maure semble entièrement construite autour du référent religieux. En effet, l'ethnoterme biạân a pour équivalent en zénaga ugudayen, sg. agadiy, terme dont l'origine semble bien être le mot arabe qădi, pl. quḍăt « juge ». De leur histoire comme « Berbères islamisés », ils n'ont retenu explicitement que la seconde caractéristique (l'islamisation).

12. D. Cohen, proposant une interprétation méliorative, m'a suggéré un rapprochement avec l'appellation signifiant «la juive », employée dans les milieux juifs les plus intégristes. Mais il faut noter que dans le cas de «la juive » (ou celui, en voie de disparition, de «la bourgeoise » dans certains milieux français modestes), le terme désigne l'épouse alors qu'en zénaga « la musulmane » s'applique à la femme en général.

13. Il est bien connu que, dans la société maure, le terme znâge s'applique exclusivement aux tributaires et j'ai montré ailleurs (C. Taine-Cheikh, 1997) que cette vision est totalement partagée par les berbérophones eux-mêmes. Notons qu'en zénaga, uẓnegen désignent les tributaires (« ceux qui s'occupent des bêtes ", hass. znâge) et le dialecte s'appelle la tuzzungiyye ou awey on uznegen (litt. « la langue des znâge ») — avec z simple spirantisé —, les zénagophones étant (litt.) « ceux de la langue des ẓnâge ». 
On imagine bien comment la constitution d'un tel idéal religieux, sous une forme aussi expressément savante (pour être juge il faut nécessairement avoir une bonne connaissance des textes en arabe classique), a pu pousser la société berbère, son élite en tête, à l'apprentissage de l'arabe littéraire ; il est possible, mais absolument pas certain, que cela les ait préparé aussi, idéologiquement, à une moindre résistance face à l'arabe dialectal ${ }^{14}$.

Par ailleurs on sait l'investissement que firent les tribus berbères de Mauritanie - comme beaucoup de convertis - dans le žihâd islamique, tant à l'époque almoravide que lors de la fameuse guerre de Šurr Bubbẹe, qui apparaît, à maints égards, comme une grande conflagration opposant principalement Arabes et Berbères. De la victoire des premiers, date officiellement, du moins dans le Sud-ouest mauritanien, le renoncement des seconds aux moyens militaires, mais les Berbères de la Gebla n'avait pas attendu ce moment pour se consacrer à l'étude du Coran.

Quant à l'arabisation dialectale elle-même, il semble difficile de ne pas voir, dans l'arrivée des Bani Hassân - et surtout dans leur(s) victoire(s) militaire(s) - , le facteur décisif de la diffusion de l'arabe comme langue usuelle et populaire de communication. Certes, l'image d'une conquête massive, par la pénétration soudaine et brutale d'un fort contingent de population, est incongrue. Dans le cas des Maures, nous n'avons affaire ni à la colonisation d'un territoire inoccupé, ni à des mécanismes de divergence ou de convergence, mais bien à un phénomène de substitution linguistique - mécanisme qui n'est pas le plus simple à expliquer, même s'il s'est souvent produit dans l'histoire des langues. L'hypothèse d'une élite dominante est naturellement celle qui convient le mieux : une minorité arabe arrive et elle finit par instaurer sa suprématie, peut-être grâce à des alliances de revers efficaces ou par une succession de victoires échelonnées sur un temps très long. En tout cas le rôle des armes semble évident puisqu'une adéquation très claire se fait, dans le dialecte zénaga comme dans le dialecte maure (klâm hassān ou hassāniyya), entre «Arabes » et "guerriers (de haut rang) » d'une part (zén. a'reb, hass. 'rab), (Bani) Hassân et 'donateurs' du dialecte arabe d'autre part (en zénaga, le dialecte hassāniyya s'appelle awey on ta'rbatt « la langue des Arabes » et les Bani Hassân sont nommés $d^{y} a^{\prime} \underline{d}$ awey on ta'rbatt « ceux de la langue arabe $\gg)$.

Pour C. Renfrew (1994), un changement linguistique imposé par une minorité nécessiterait une organisation centralisée et un grand prestige de la langue. Ceci est à nuancer dans le cas du klâm hassān mais on imagine bien que le dialecte a profité du prestige de l'arabe littéraire et que la hiérarchisation tribale, autour des centres constitués par les émirats, répond peut-être, d'une certaine manière, à la condition requise. Un dernier problème reste à résoudre,

14. Il peut être utile de dissocier l'acquisition du hassāniyya par les berbérophones et la perte du zénaga. En effet, je pense probable l'existence d'une phase de bilinguisme éventuellement fort longue. Par ailleurs, continuer à parler le zénaga ne signifie pas forcément qu'on refuse de parler le hassāniyya, surtout si le dialecte arabe remplit des fonctions de communication importantes. Si l'on observe une meilleure conservation du zénaga chez les zwâye (du moins certains d'entre eux, peutêtre moins proches géographiquement et socialement des Hassân), c'est sans doute parce qu'ils avaient du berbère une pratique très développée, y compris dans l'enseignement et dans la poésie. 
celui de la grande homogénéité du hassāniyya actuel, pas facilement compatible, à priori, avec la diffusion à partir de plusieurs centres distincts. Pour cette raison il semble particulièrement difficile de minimiser le rôle décisif des Bani Hassân en tant que groupe, mais je voudrais aussi attirer l'attention sur le rôle complémentaire probable des migrations qui semblent avoir marqué de tout temps les histoires individuelles, tout comme les migrations-recompositions ont marqué celles des tribus.

D'une certaine manière le hassāniyya s'est peut-être constitué partiellement comme une koinè, écartant les particularismes trop marqués de groupes arabophones de diverses origines, mais il n'a sans doute pu le faire que grâce au prestige des Bani Hassān (litt. «les fils de Hassan ») et, symboliquement, au nom de leur père Hassān - ce qui transparaît dans la locution klâm hassān qui, littéralement, signifie « parler de Hassan ».

\section{Le règne de l'auralité}

Même si la société des biđận, aux $18^{\mathrm{e}}$ et $19^{\mathrm{e}}$ siècle, n'est pas encore complètement arabisée, il semble que cette période corresponde à un certain " âge d'or » de la culture maure traditionnelle, où dialecte hassāniyya et arabe littéraire entretiennent des rapports bien circonscrits mais complexes, à la fois entre eux et avec les forces sociales en présence.

\section{La langue du Coran et les mots du dialecte}

Dans le champ du savoir (celui des lettrés qui tourne tout entier autour du Coran et des sciences islamiques), ce qu'on appelle couramment l'arabe littéraire joue un rôle fondamental. Si l'on prend d'ailleurs « à la racine » les deux termes «littéraire » et «lettrés », toute la culture savante du monde arabe serait basée sur la lettre - autre façon sans doute de dire qu'elle se fonde sur l'écriture. Pourtant celle-ci n'a pas accédé, pour les Arabes eux-mêmes, au statut de référent ultime, de garant suprême et il est sans doute nécessaire d'oublier le pouvoir que l'écrit a acquis dans la culture occidentale pour sortir de la fausse opposition oral / écrit.

Pour C. Herrenschmidt (1998), la naissance de l'écriture dans l'Orient antique est profondément liée à la religion et les documents comptables euxmêmes - dont l'importance a été soulignée par J. Goody —, ne jouaient un rôle central que parce qu'ils concernaient les comptes que les humains entretenaient avec leurs dieux. Ce lien premier de l'écriture avec l'invisible ${ }^{15}$, beaucoup de cultures l'ont préservé, notamment celles qui s'en sont tenus à des alphabets consonantiques. Si les voyelles ne sont pas marquées, le texte a besoin du corps de l'homme, de son souffle et de son esprit, pour se matérialiser. Alors que

15. «L'écriture atteste la piété envers les dieux, prolonge le rite et les rend perpétuels. [...] Dans l'écriture, ces deux invisibles que sont le langage et les dieux sont présents, visibles, immobiles, connaissables ». (idem : 117). 
l'alphabet incomplet impose une lecture-compréhension « en prophète » (la saisie du mot précédant la voyellation et la syllabation), l'alphabet complet, comparable en lui-même à la parole, dénoue le lien entre lecture et compréhension, de sorte qu'on peut lire sans comprendre.

S'il y a bien, historiquement, un rapport solidaire très fort entre le monothéisme et l'écriture consonantique, il y a aussi, dans la culture musulmane, un rejet particulier de l'image qui redouble la condamnation de la voyelle. On dira donc, en reprenant la conclusion de $\mathrm{A}$. H. Ibrahim à son étude du système d'écriture de l'arabe : «pendant longtemps l'histoire du rapport des Arabes à leur langue est aussi celle de leur résistance à sa transcription » (1990:228). Dans la mesure où cette résistance s'est menée au nom même de la religion, il est assez curieux, d'un certain point de vue, que le texte du Coran soit celui pour lequel le marquage des voyelles est le plus fréquent. A. Bounfour (1994b) nous propose cependant, pour la première voyellation du Coran, un récit d'origine qui montre bien toutes les précautions qui furent prises par le maître al-Du'alî (couleur différente pour les voyelles, tracé de celle-ci par un disciple, ...) pour obéir au gouverneur omeyyade sans que sa voix perde le contrôle des consonnes ${ }^{16}$.

Le risque de fautes (le lahn) est bien sûr au centre du problème et le souci de les éviter semble particulièrement développé chez les zwâye maures car une erreur publique peut mettre en jeu leur honneur de lettré17. Il est possible cependant qu'ils aient résisté plus que d'autres à la tentation de voyeller puisque, même dans l'écriture des noms propres, ils rejettent l'usage des voyelles et recourent à une formule descriptive malcommode et peu commune ${ }^{18}$. Par contre, ils semblent avoir poussé assez loin l'apprentissage de la grammaire comme remède à cette menace. Dans les biographies des plus grands lettrés, on trouve souvent un épisode initial « fondateur » où une résistance première tombe après un lahn ayant suscité les rires moqueurs du maître ou de l'assistance (cas notamment du fameux prosateur Šayh Sîdi Muhammad ou du plus grand grammairien maure, Muhtâr W. Bûna).

On a souvent relevé le lien entre le nom du Coran (Qur'ān) et le sens de « récitation » - lien sur lequel je reviendrai —, mais je voudrais souligner ici le sémantisme du verbe gra qui, en hassāniyya, signifie certes « lire », comme le cl. qara'a, mais aussi, très clairement, " étudier ». Lire est donc d'emblée considéré comme un phénomène complexe, qui nécessite des aides spécifiques. J'ai déjà évoqué le cas de la voyellation, celui de la grammaire (plus généralement, on peut parler des diverses sciences étudiées par le faqîh), mais on peut ajouter un autre adjuvant extrêmement important, celui du dialecte. C'est en effet en hassāniyya que se donnent les explications et que se font les commentaires ; aussi peut-on dire que le dialecte arabe a hérité dans la société maure du rôle de

16. «...ce n'est pas lui qui exécute cette voyellation mais un disciple. Il reste le maître de la voix comme le fut le Prophète et ne lit que des consonnes. La voyelle doit rester invisible à son regard et il est interdit à sa main de la tracer » (1994:125).

17. Sur le problème du lahn en poésie, cf. A.-B. Miské, $1970: 47$.

18. Solution dont s'étonnait récemment devant moi U. Rebstock, spécialiste des manuscrits arabes et en particulier de ceux de Mauritanie. 
«langue d'enseignement» dont jouissait auparavant le zénaga ${ }^{19}$. L'existence d'une traduction versifiée, en dialecte, du fameux ouvrage de grammaire intitulé Ajarrûm, est sans doute la meilleure preuve qu'on puisse donner de la complémentarité traditionnelle du dialecte et de l'arabe classique dans l'enseignement traditionnel.

\section{La bouche du griot et l'oreille du marabout}

Dans le cas du Coran, il est bien clair qu'on a affaire, à l'origine, à de la pure oralité et à la supériorité indiscutable du témoin oral sur le témoignage écrit (Ibrahim, idem : 227). Cependant, quand le temps du témoignage oral direct est révolu — d'où la nécessité du Livre - la complémentarité de la lettre et de la voix s'instaure. Elle finit par conférer un nouveau statut à la langue du Coran et la pratique de l'arabe « classique » devient celle d'un écrit oralisé.

La récitation du Coran, pierre de touche de l'enseignement, est certes orale et le sémantisme du verbe hafiạa (hass. hfaḍ) souligne bien le lien indissoluble entre «réciter (le Coran)» et «conserver », mais elle n'est aucunement coupée de l'ordre scriptural et du phénomène de la lecture. Par contre la place de celle-ci s'inscrit entièrement dans l'oralité, ce qui contribue puissamment à marquer la pratique de cet arabe classique. On remarquera tout d'abord que l'apprentissage de la lecture est toujours second : par rapport à l'écriture puisqu'il s'agit d'abord d'apprendre les lettres ; par rapport à la récitation puisque l'enfant ne lit que ce qu'il a d'abord récité. Enfin on rappellera que, même quand elle cesse d'être une lecture-récitation, la lecture à voix haute est restée le modèle traditionnel prototypique.

Entre la lettre et la voix, donc l'œil et la bouche, l'arabe «littéraire » semble chercher désespérément son unité et, par là même, son statut. Comment définir son oralité ? Comment la situer par rapport à celle des sociétés dites « sans écriture »?

Pour moi, l'oralité est effectivement secondaire mais par rapport à ce que je propose d'appeler l'auralité, c'est-à-dire un ordre discursif qui transcende l'opposition oral / écrit et met l'accent sur l'aural. C'est par l'audition ${ }^{20}$ que le jeune est éduqué et c'est parce que l'oreille joue un si grand rôle dans l'enseignement que les textes les plus scientifiques sont mis en vers - non pas par volonté de charmer (la métrique de ces textes diffère de celle de la véritable poésie) mais parce que la mesure permet à un texte entendu de mieux s'imprimer dans la mémoire, surtout si le balancement du corps, accompagnant la psalmodie de la récitation, sert de métronome au rythme du vers.

Ce rôle primordial de l'audition dans l'enseignement traditionnel fait des lettrés, et plus généralement des zwâye, les grands spécialistes de l'auralité. Or il se trouve que ceux-ci, dans l'imaginaire social, sont représentés comme dotés de

19. Pour un emploi comparable du bambara, cf. l'étude précise de T. Tamarin (1996).

20. C. Fortier (1997) a bien décrit l'importance de la mémorisation et de l'audition dans l'enseignement traditionnel. Cependant, mettre en avant l'audition permet peut-être de dépasser l'opposition écrit / oral dont on ne sort pas par un simple renversement oral / écrit. 
grandes oreilles. On a suggéré que le lettré devrait le développement de cet appendice au fait qu'il était considéré comme couard, à l'instar du lapin (et par opposition, bien sûr au courageux guerrier), j'y verrais plutôt l'emblème de sa spécialisation et le signe qu'il est bien équipé pour « entendre » la Parole divine et ouvrir son âme à la connaissance.

Pour trouver la véritable oralité, il faut se tourner vers le griot. C'est lui qu'on peut considérer comme le maître de la parole, celui qui fait et défait les réputations en général, mais dont la fonction est étroitement liée au groupe des guerriers et notamment au pouvoir émiral.

Si l'on distingue le theydîn du gne ${ }^{21}$, on dira que la première - la poésie à accents épiques des griots - est née avec Saddûm W. Ndjartu au $18^{\mathrm{e}}$ siècle. Or on peut noter que, dans les éléments biographiques recueillis par A.W. O. Cheikh, l'épisode fondateur est la visite qu'un envoyé divin lui aurait faite pendant son sommeil : en lui crachant dans la bouche, il aurait fait de Saddûm le prince des griots 22 .

D'un point de vue métrique, il existe un lien assez clair entre le theydîn du griot et la poésie classique ancienne dont les lettrés maures furent parmi les derniers à perpétuer le modèle vivant (C. Taine-Cheikh, 1995 : 195-6). Rival bien connu du marabout, le griot n'a en fait, officiellement, ni le même maître, ni les mêmes armes que lui. Tandis que le premier manie l'arabe littéraire - en prose ou en poésie - pour la plus grande gloire de Dieu, le second compose des poèmes en dialecte pour magnifier les vertus du preux combattant ${ }^{23}$. Il existe un cas d'interaction entre les deux univers - l'imitation par un lettré, Muhammad al-Yadâli (mort en 1757), d'un poème (un rașm) composé pour un prince et la fureur de ce dernier face à ce qu'il considéra comme un vol - mais cet exemple montre parfaitement les limites d'une telle intéraction. D'une part le lettré fut immédiatement pardonné quand il expliqua qu'il avait «détourné » le poème pour la gloire du Prophète, leur supérieur à tous. D'autre part on peut constater que l'imitation concerne fondamentalement la métrique et la prosodie mais non la langue, puisque le poème du lettré est en arabe classique et le rașm, en dialecte $^{24}$. Si l'arabe littéraire est lié significativement à l'oreille du marabout, l'arabe dialectal peut donc être associé à la bouche du griot.

21. Cet autre type de poésie en dialecte, dont les musiciens-chanteurs n'ont pas l'exclusivité, a d'autres fonctions et des caractéristiques formelles sensiblement différentes (C. Taine-Cheikh, 1994a), qui semblent plus influencées par la poésie andalouse.

22. Le griot est pris ici comme figure emblématique de la culture en dialecte à cause de ses liens avec le gine en général et avec le theydîn en particulier, mais la culture orale ne s'identifie pas au groupe des griots comme la culture aurale, à celui des zwâye. Pour avoir une vue plus large de l'oralité dans une culture comparable, à bien des égards, à celles des Maures en hassāniyya, on pourra consulter l'article de J. Drouin sur l'esthétique de la communication verbale chez les Touaregs (1987).

23. Pour une analyse de ces vertus et, plus généralement, pour une étude précise des rapports entre pouvoir et émiral et griots, cf. A.W. O. Cheikh (1985: 407 et sq.).

24. Même dans le cas du célèbre raṣm de Saddûm, à la langue fort obscure, la base lexicale et morpho-syntaxique reste incontestablement dialectale. 


\section{Les tentatives du saint ou le vertige de l'écriture}

Par rapport à l'écrit, j'ai insisté sur le phénomène de la lecture et sur son lien avec la récitation. J'ai évoqué aussi l'aspect conservatoire de l'écriture qui est à l'origine du Coran comme texte et qui a motivé le développement des sciences islamiques. Aussi n'est-il pas forcément utile de revenir sur le rôle de la copie et de la répétition dans cette société traditionnelle où les lettrés ont développé, selon les termes d'A.W. O. Cheikh, une « culture de ruminants».

Dans cette optique où la bid'a, l'innovation, est synonyme d'hérésie, la notion d'auteur - du moins en dehors de la poésie - n'a certainement pas les mêmes contours que dans la conception moderne occidentale. Je souhaiterais cependant étudier le cas de quelques lettrés dont les noms, restés gravés dans les mémoires, sont associés à des ouvrages considérés comme importants 25 .

On considère que le plus ancien texte mauritanien connu qui nous soit parvenu date du $11^{\mathrm{e}}$ siècle, mais il est dû à un Grenadin (ou considéré comme tel malgré son origine kairouanaise), al-Murādī al-Haḍramī al-Qayrawānī, second prédicateur du mouvement almoravide. Ce livre, intitulé Kitāb al-išāra ilā adab alimāra, se présente comme un «Miroir du prince» sans qu'on sache s'il fut vraiment composé pour l'éducation d'un futur homme de pouvoir. L'ouvrage rappelle des écrits d'inspiration orientale, tel celui de Kalîla wa Dimna, auxquels fait peut-être référence l'expression qālat al-hukamā' « les sages ont dit" 26 .

Alors que le personnage d'al-Murādī s'inscrit dans une tradition théologique rationalisante (la tradition du kalâm, rare au Maghreb et qui s'achève, semble-t-il, avec lui), il réapparaît curieusement plusieurs siècles plus tard en association avec un courant de pensée dont il semblait a priori fort éloigné. D'une manière générale, en effet, il existe différents courants chez les lettrés qui tendent à se cristalliser autour de deux figures islamiques, le faqîh et le walî, sans que l'une et l'autre soient en fait radicalement incompatibles ${ }^{27}$.

Parmi les polémiques les plus célèbres, on peut en compter au moins trois qui tournent autour du problème des miracles et concernent de purs fuqāha, particulièrement réputés comme grammairiens. Dans le cas de W. Billa'maš, mort en 1694 - premier auteur d'envergure et disciple de W. Bū-1-Muhtâr (qui polémiqua contre Nașr ad-Dîn et l'idée d'un Etat musulman) -, on peut dire que son adversaire chercha appui auprès d'al-Murādī al-Haḍramì lui-même. Six siècles après la mort du prédicateur almoravide, celui qui allait rester à la postérité sous le surnom d'imâm al-Majdûub (litt. «l'inspiré ») redécouvrait la

25. Pour tout ce qui concerne l'imâm el-Haḍramī, je précise tout de suite que je me suis largement (et librement) inspirée de l'article de A.W. O. Cheikh et B. Saison (1987).

26. Cette formule qui, avec son répondant 'aqūlu 'inna «je dis que», ponctue largement le texte, prouve - si besoin est - que le lexique du discours écrit reprend largement celui de l'oral.

27. Dans le détail des multiples controverses qui ponctuent l'histoire intellectuelle de la société maure, la situation se complique du fait que trois grands mouvements se partagent le champ confrérique (la šadilìya, la qādirìya et la tijāniya). Elle se complique plus encore autour de la définition du fiqh. Si beaucoup, en effet, condamnent le kalâm (mais Muhammad al-Yadâli, par exemple, ne se prononce pas) et si quelques-uns sont à la fois contre le kalâm et la logique, seul un petit nombre, considéré comme des fondamentalistes, rejette aussi les usūul (les «fondements ») du fiqh. 
tombe d'al-Hadramì. Plus encore, son bras droit allait soudain se mettre à enfler et il ne retrouverait sa forme première qu'après avoir écrit, comme sous la dictée du mort, le Kitâb al-Minna, « Le livre de la Grâce ».

Dans l'histoire de la société maure, ce livre pourrait être considéré, d'une certaine façon, comme un premier exemple de texte écrit dont la conception se situe en dehors du domaine de l'auralité. Le fait cependant que l'écriture « automatique ${ }^{28}$ de ce livre soit aussi une version transposée de la prophétie de Muhammad, montre les limites de l'événement et souligne d'autant la mise en tutelle de l'écriture.

De la controverse entre Šayh Sîd al-Muhtâr al-Kûnti et le grand grammairien Muhtâr W. Bûna, on retiendra que le premier, qui défendit les miracles ${ }^{29}$, fut, entre autres, un infatigable épistolier, donc l'un des lettrés maures qui recourut le plus, peut-être, à la communication écrite. Quant au fils de Šayh Sîd al-Muhtâr († 1826) dont l'œuvre est consacrée à la biographie de ses parents, il est considéré comme le premier grand prosateur maure.

Ce pointage de quelques écrits marquants de la culture maure ne visait qu'à une chose, montrer les cas-limites où l'écriture donnait les signes ténues d'un affranchissement par rapport à l'auralité. Il m'a semblé que, dans ce mouvement d'autonomisation, le mysticisme, à travers la figure du wali, jouait un certain rôle.

\section{Une laïcisation difficile}

Jusqu'à la colonisation et les bouleversements qui s'en suivront pour la société maure, on peut donc dire que les deux groupes dominants n'ont pas exactement les mêmes pratiques linguistiques. Alors que le pouvoir des guerriers est lié au dialecte hassāniyya par l'intermédiaire des griots, celui des lettrés passe fondamentalement par l'usage de l'arabe littéraire sous sa forme particulière d'écrit oralisé (ou aural). Au $20^{\mathrm{e}}$ siècle, cependant, la situation est petit à petit remise en question.

\section{Les petites échappées linguistiques ou les vertus de la citation}

Au tournant du $20^{\mathrm{e}}$ siècle, l'installation d'un lettré maure au Caire est à l'origine d'une grande fresque intellectuelle. Etant arrivé dans un milieu de savants pour lesquels les habitants du lointain Sahara occidental étaient souvent vus comme des demi-arabes à la culture réduite, les circonstances l'amenèrent à témoigner du contraire. Sid-aḥmad W. Alamîn était un homme fait quand il quitta son pays natal, aussi était-il en mesure de faire un tableau précis de sa société et de son histoire. La partie la plus importante de l'ouvrage est cependant

\footnotetext{
28. L'imâm al-Majdûb étant réputé analphabète, son bras aurait écrit sous l'emprise directe de l'esprit de l'imâm al-Ḥaḍāmi, sans que la part consciente de l'écrivant intervienne.

29. Il fut à la fois un spécialiste du fiqh et un mystique - c'est le premier et principal propagateur de la confrérie qādirìya au Sahara.
} 
occupée par une anthologie des poètes maures. C'est d'ailleurs parce qu'il put réciter de mémoire des milliers de vers (4500) que W. Alamîn suscita l'intérêt et l'admiration de ses interlocuteurs orientaux pour la culture de son pays.

Cette anthologie, intitulée $A l$ wasit fì taräjimi 'udabā'i Šinqịt (« Le meilleur [livre] sur les œuvres des poètes et hommes de lettres du Shinqît », selon la traduction d'A.-B. Miské), reste une référence majeure pour le ši $r$, la poésie en arabe classique mais on notera que la poésie en dialecte n'y a pas sa place - tant le gne proprement dit que la poésie des griots. Il s'agissait en effet de mettre en avant la «pureté » de la langue littéraire telle que la pratiquaient les lettrés maures et, pour témoigner de la perfection et de la beauté de cet arabe, rien n'était plus approprié que le šír composé selon les canons quasi-immuables de la qașida, censés reproduire ceux de la poésie anté-islamique ${ }^{30}$.

Pourtant, quelques lettrés vont bientôt remettre en cause ce modèle contraignant qui rend toute véritable spontanéité impossible. C'est à Mhammad W. Ahmad Yûra qu'on attribue l'initiative d'avoir introduit dans le šír des éléments empruntés au dialecte - en général des toponymes, expressions imagées ou proverbes qui sont là pour faire signe, ancrer le poème dans l'univers plus intime et plus subjectif que seul le dialecte réussit à exprimer. Cette souplesse que permet le hassāniyya, les lettrés en sont conscients car, s'ils furent un peu réticents à pratiquer le gne à ses débuts, du moins finirent-ils par y exceller. Dans le cas de W. Ahmad Yûra, on notera avec intérêt qu'il s'agit d'un vrai magicien des mots, puisqu'il était poète en arabe classique, en hassāniyya et en zénaga. Peut-être son bilinguisme originel est-il d'ailleurs pour quelque chose dans sa sensibilité linguistique. En tout cas ce qu'on appelle la « bigarrure » de W. Aḥmad Yûra ne passa pas inaperçu dans l'histoire de la poésie maure.

Il serait intéressant de savoir si le mouvement inverse - qui consiste à introduire des citations d'arabe littéraire (vers, formules coraniques, etc.) dans des poèmes en dialectal - est contemporain du précédent. Dans la poésie féminine (où le phénomène est fréquemment attesté), certains croient plutôt à une tendance récente qui serait née du désir de donner un air plus sérieux, voire plus religieux, à une poésie dont la légitimité reste contestée. Pour Miské, il faut songer au rôle de pionnier d'un homme comme Hammâm Fall qui s'est fait connaître entre autres par des emplois parodiques du ši ${ }^{i}{ }^{31}$. Mais on peut aussi imaginer que l'innovation n'est entrée dans la poésie que dans un second temps, après avoir été d'abord exploitée dans des genres moins formels. Il est en effet curieux de constater que, parmi les contes recueillis par R. Basset au début du siècle, on trouve six historiettes d'un genre tout à fait original, où toute la saveur du récit repose sur l'insertion d'une citation coranique et sur le fait que, derrière

30. Il est symptomatique que $b d a^{\varsigma}$, «innover », signifie également «composer, improviser des vers » mais uniquement en ce qui concerne la poésie en ḥassāniyya.

31. «En utilisant la prosodie de la poésie classique, il y met un 'contenu' tout à fait inhabituel : mots hassaniya 'arabisés' d'une manière comique, parodie des règles grammaticales, etc. L'ensemble (une alliance contre nature) est souvent d'un effet irrésistible » (Miské, $1970: 63$ ). 
le sens premier fournit par la langue littéraire, se cache un autre sens, amené par le contexte, mais illégitime puisqu'emprunté au dialecte ${ }^{32}$.

Nous voyons donc se dessiner, dans un monde encore complètement dominé par la diglossie, diverses tentatives pour faire que les deux variétés d'arabe se rencontrent et se fertilisent mutuellement. Tandis que, dans le monde profane du dialecte, surgit la langue du sacré (sur le mode sérieux de la morale ou sur le mode transgresseur de la parodie), dans le monde désincarné de l'arabe littéraire perce le sentiment sous les mots du dialecte.

\section{Les grandes échappées linguistiques ou les problèmes de la trahison}

Face à la variété dialectale qui sert à la fois aux communications courantes et comme langue de l'intimité, de l'affection et de l'humour, l'arabe littéraire incarne, à n'en pas douter, la langue de la religion et de la science (il s'agit bien sûr des sciences islamiques) - d'où le proverbe al-‘ilm, mā vî-h saḥwe «il n'y a pas de pudeur en science » qui exprime en dialecte la possibilité de recourir à l'arabe classique pour traiter de problèmes délicats (la sexualité par exemple) sans tomber dans la grossièreté. Au $20^{\mathrm{e}}$ siècle, d'après les témoignages qu'on peut avoir de situations « diglossiques » comparables, la langue littéraire s'opposerait plutôt au dialecte comme la langue de la raison à la langue des sentiments ${ }^{33}$. C'est en fait dans cette distance instaurée entre la langue de la science et celle de la raison que le français va se glisser et venir menacer, plusieurs décennies durant, la position dominante de l'arabe littéraire (C. TaineCheikh, 1994b).

Je ne referai pas ici l'histoire de la scolarisation durant la période coloniale, même si la position du français au moment de l'Indépendance ne peut se comprendre en dehors d'elle. On sait que l'imposition de la langue des colonisateurs ne fut jamais vraiment du goût des populations mais que certaines surent et purent mieux résister que d'autres, de telle sorte que la langue française apparut, dans les années 60 , comme inégalement représentée, d'abord par rapport à la population mauritanienne en général, mais aussi par rapport à la population maure en particulier.

Le combat que menèrent les élites contre la scolarisation en français se fit très clairement au nom de l'arabe comme langue de culture et de religion, mais la colonisation fit toujours la distinction entre les deux et consentit plus de concession envers la première qu'envers la seconde - d'où le refus beaucoup plus net qu'elle opposa pour l'enseignement de l'arabe aux Négro-Africains.

32. «L'HOMME ET LA FEMME ENCEINTE. Une nuit un homme méchant marchait et trouva une femme couchée en dehors de la tente : elle était enceinte. Il lui dit : Lève-toi! Lève-toi tout de suite de là. - Pourquoi ? demanda-t-elle — Dieu n'a-t-il pas dit : Et il maîtrisa son âme dans ses penchants. [Note : Qorân, Sour. LXXIX, 40. Jeu de mots sur n.f.s. « enceinte » en hassaniya et n.f.s. «âme » et entre h.w.y. " penchants » et h.w.y. «air »] (R. Basset, $1913: 657)$.

33. Je pense notamment au cas de l'Italie avec l'interview de l'écrivain sicilien Andrea Camilleri par M. Abescat dans Le Monde ou le choix du dialectal Amarcord fait par le cinéaste F. Fellini quand il s'agit de parler de son enfance. 
Les enfants qui fréquentèrent l'école publique avant la fin des années 70 reçurent donc une formation en français de type laïque, même s'ils continuèrent, à d'autres moments de la journée ou de l'année (le soir et pendant les vacances), à suivre l'enseignement traditionnel de l'école coranique, généralement commencé avant l'entrée à l'école « moderne». Beaucoup ne furent pas insensibles au gouffre béant qui s'étendait entre les deux types d'enseignement, véhicules de deux cultures différentes et de deux modes de pensée fondamentalement divergents. Pour ceux, longtemps peu nombreux, qui se sentirent appelés (voire happés) par la culture occidentale, le Centre culturel français St-Exupéry fut bien souvent le seul lieu où l'on pouvait réellement entrer en contact avec un monde étranger (et étrange) qui, par ailleurs, restait très scolaire. Si l'on pense à la bibliothèque de ce centre et à l'extrême difficulté qu'elle avait à récupérer les livres qu'elles prêtaient, on comprend que les jeunes lecteurs apprirent vite à distinguer les deux univers culturels. Alors que l'écrit faisait, dans la société maure, l'objet d'une attitude respectueuse très marquée et quasi sacrale (il faut manipuler l'écrit avec un grand soin, on n'enjambe pas un manuscrit, on ne verse pas n'importe où l'eau de rinçage de la planchette qui a servi à l'écriture du Coran, ...), les livres imprimés en français avaient une survie difficile et un parcours imprévisible, preuves qu'ils n'attiraient aucune marque de respect en tant qu'écrit.

Cette différence de traitement selon la langue employée se retrouve à divers niveaux et notamment celui de la forme du contenu. Il est significatif par exemple qu'un texte en arabe (y compris dans les revues scientifiques) doive toujours être introduit par une invocation du nom d'Allah ${ }^{34}$.

Derrière le choix entre l'arabe littéraire et le français (il en serait de même, bien sûr, avec l'anglais), il y a donc d'autres choix qui se profilent presque automatiquement : des moyens linguistiques plus ou moins adaptés à l'emploi langagier qu'on souhaite (un bilingue peut, par exemple, préférer le français pour la prose et l'arabe pour la poésie) ; des champs discursifs régis par des règles différentes (statut hétérogène de l'auteur et de la citation, rôle divergent de l'argument d'autorité, ...) et contrôlés par des instances sociales plus ou moins conservatrices ; un public plus ou moins réceptif à certains types de propos (pour une remise en cause de l'idéologie dominante, la langue étrangère est souhaitable, à la fois moins dangereuse et plus efficace car elle laisse à l'écart ceux qui ne pourraient accepter certaines critiques).

Linguistiquement et socialement, on n'écrit pas la même chose en français et en arabe littéraire, mais c'est aussi la raison qui fait que, pour beaucoup de Mauritaniens, la pratique assidue du français passe facilement pour une forme de trahison nationale.

34. Je parle là au nom de mon expérience personnelle, en particulier celle de secrétaire de rédaction d'Al-Wasit, la revue bilingue de l'Institut Mauritanien de Recherche Scientifique. 


\section{Le mariage de raison ou la difficulté du mélange}

C'est avec le développement du nationalisme dans le monde arabe et sous l'influence de la radio que, dès la fin des années 60, le sentiment de la jeunesse arabophone mauritanienne développa un rejet plus marqué du français, du moins en tant que langue prépondérante. Mais, dans le même temps, elle se fit la propagatrice d'une nouvelle variété d'arabe qui cherchait à cumuler les avantages (en évitant les inconvénients) des deux variétés pratiquées jusqu'à présent.

Parce qu'elle empruntait à l'arabe littéraire son vocabulaire abstrait et ses constructions syntaxiques complexes et conservait, du dialecte, le lexique de base et l'essentiel de la morphologie (une morphologie qui paraît d'autant plus simple aux hassanophones qu'elle leur est parfaitement familière), cette variété fut perçue comme une variété mixte, occupant une position proprement intermédiaire entre les deux pôles précédemment identifiés. Le nom d'« arabe médian » qui lui fut donné, notamment en Mauritanie (comme équivalent d'al'arabiya l-wușta «l'arabe du milieu»), est particulièrement mérité dans le domaine des réalisations phonétiques et de la structuration syllabique puisque, selon l'origine de chaque lexème et selon même, parfois, le contexte dans lequel il s'insère, certains phonèmes consonantiques changent de réalisation $(g / q, d$ / đ, ...) et les voyelles brèves se maintiennent ou non.

Aux yeux des lettrés traditionnels, cependant, cette nouvelle variété d'arabe apparut comme proprement monstreuse. Il y avait certes des problèmes de vocabulaire qui pouvaient désorienter les anciens, tel celui de $d$-dowle qui était connu en dialecte avec le sens de «groupe d'étudiants rassemblés ; grand troupeau » et prenait, sous l'influence de l'arabe littéraire, le sens de «Etat, nation ». Mais, fondamentalement, le rejet était plus profond car il relevait d'une sorte de tabou, celui qui veut qu'on ne fasse pas de mélange contre-nature.

Dans les cas évoqués précédemment (cf. 3.1.) et en particulier dans celui de la zreyge de W. Aḥmad Yûra, il s'agissait littéralement d'une " bigarrure », c'est-à-dire d'un assemblage de pièces disparates, le poète juxtaposant des éléments d'origine différente sans pour autant chercher à supprimer les différences ou à effacer les frontières entre les blocs ${ }^{35}$. Avec l'arabe médian, les guillemets de la citation sont en quelque sorte supprimés (pour autant qu'on puisse parler, à l'oral, de guillemets) et l'on se retrouve avec des formes hybrides qu'on ne peut plus classer.

À sa manière, ce que l'arabe médian reproduit, c'est un mélange des genres comme celui que réalise l'hermaphrodite, cet être mi-homme mi-femme qui pose tant de problème en terre islamique - et pas seulement parce que les calculs de sa part d'héritage y sont éminemment compliqués. Même sans pousser la comparaison aussi loin, on peut remarquer l'importance que la société maure traditionnelle (suivant en cela des traditions arabes, voire sémites, très anciennes) accorde à ce problème du mélange, que ça soit dans sa diététique (où

35. Le fait que le changement de variété se produise généralement en finale (comme dans l'historiette recueillie par R. Basset) montre bien que la place est significative et le bornage, très apparent. 
elle proscrit l'association d'aliments comparables telles le lait et la viande) ou dans sa conception de la filiation (laisser les généalogies se mélanger est un pêché et le texte généalogique - la nusha, litt. «copie» -, est là pour qu'on puisse s'en prémunir).

Face à l'arabe médian qui pouvait apparaître avec quelque raison comme une solution judicieuse pour moderniser tout à la fois l'arabe classique et le dialecte, on peut donc dire que les forces traditionnelles ont montré très peu d'enthousiasme. Cela ne signifie pas, bien sûr, que cette variété d'arabe n'a pas continué son existence mais seulement qu'elle n'a pas suscité un grand intérêt de la part des pédagogues et n'a donc eu qu'un impact très limité sur les méthodes de l'enseignement.

On peut donc se demander si les progrès de la scolarisation en arabe, très importants depuis les années 80 , a permis vraiment de sortir de l'auralité sous sa forme la plus stéréotypée et la plus limitée. On aurait aimé, en particulier, avoir l'assurance que les petits écoliers mauritaniens d'aujourd'hui ont cessé d'apprendre (par cœur) pour lire et qu'ils ont la possibilité de lire pour apprendre (sur ce dilemne, cf. Ibrahim, 1994). Mais pour cela il faudrait sans doute que l'arabe écrit et l'arabe oral (moderne) se libèrent l'un de l'autre et qu'ils cessent de se surveiller mutuellement, au nom de l'arabe classique sanctifié par l'islam.

A certains égards, la société maure connaît de grands changements, mais à bien d'autres, elle semble souvent faire un pas en avant et deux pas en arrière.

Après l'Indépendance, les forces vives du pays voulaient s'ouvrir au monde moderne occidental par le français et/ou se fondre dans la nation arabe en développant une pratique modernisée et simplifiée de l'arabe littéraire ; le conflit qui en résulta semble avoir abouti à un échec pour l'une et l'autre tendance. Il y eut un temps, au début des années 90 notamment, où la floraison des journaux indépendants - d'abord en français, puis en arabe également - fut le signe d'un éveil intéressant de la production écrite et on put alors imaginer que le déclin manifeste de la poésie orale (lié au changement de mode de vie et de repères culturels) serait bientôt compensé par un développement de la littérature écrite. Actuellement la transformation des élites semble marquer le pas et il est assez difficile de faire des pronostics ${ }^{36}$, même si l'on voit bien que le fond culturel bédouin - et donc le dialecte sous ses formes les plus riches et les plus prestigieuses - est gravement menacé. Du moins peut-on espérer que, du « travail du négatif » sorte quelque chose de positif, un ou plusieurs outils linguistiques souples et efficaces pour répondre à tous les besoins. Ce sont là des accouchements qui demandent du temps, mais sans doute moins que celui qui consacra le remplacement du zénaga par le hassāniyya. Quant à la religion, elle n'a sûrement pas dit son dernier mot et je crois qu'elle continuera à jouer son rôle dans les changements linguistiques, que ce soit directement, par le contrôle

36. Il serait intéressant de pouvoir mesurer l'impact des télévisions étrangères, en particulier celui des télévisions arabes qui parviennent très facilement par satellite depuis quelques années. On n'a pas encore oublié à Nouakchott le succès incroyable de Guadalupe, le «Dallas » brésilien, doublé par des Libanais et diffusé par la chaîne saoudienne MBC émettant de Londres ! 
qu'elle exerce sur l'arabe littéraire, ou indirectement, par l'influence qu'elle a sur la représentation hiérarchisée des productions langagières.

\section{Bibliographie}

ABROUS, D. (1992), «Les joutes poétiques du henné : Compétition d'honneur et rapt symbolique », E.D.B., 9, 147-164.

BASSET, R. (1913), Recherches historiques sur les Maures, in Mission au Sénégal, Paris : Leroux, 435-665.

BONTE, P. (1998), L'émirat de l'Adrar. Histoire et anthropologie d'une société tribale du Sahara occidental, Thèse de Doctorat d'Etat, EHESS.

BOUNFOUR, A. (1994a), Le næud de la langue - Langue, littérature et société au Maghreb, Aix-en-Pr. : Edisud.

- (1994b), «L'écrit parlant \& l'écrit silencieux. Le problème de la transcription arabe de la voyelle berbère », E.D.B., 11, 123-132.

CAMPS, G. (1983), «Comment la Berbérie est devenue le Maghreb arabe », R.O.M.M., 35, 7-23.

CLAUDOT-HAWAD, H. (1993), Les Touaregs. Portrait en fragments, Aix-en-Pr. : Edisud.

- (1996), « Touaregs: l'identité en marche»,E.D.B., 14, 223-232.

CORTADE, Fr. J.-M. \& M. MAMMERI (1967), Lexique français-touareg, Alger : A. M. G.

CUOQ, J. (1974), Recueil de sources arabes concernant l'Afrique Occidentale du VIIe au XVe siècle, Paris, CNRS.

DROUIN, J. (1987), « De quelques conceptions esthétiques de la parole dans la société touarègue », Journal des Africanistes, 57 / 1-2, 77-95.

FORTIER, C. (1997), «Mémorisation et audition : L'enseignement coranique chez les maures de Mauritanie », I.S.S.S., 11, 85-105.

GALAND, L. (1981), «Variations sur des thèmes berbères en $d^{\prime}$ », C. r. du G.L.E.C.S. (1973-79), XVIIIXXIII / 2, 311-20.

HERRENSCHMIDT, C. (1998), L'écriture entre mondes visible et invisible en Iran, en Israël et en Grèce, in L'orient ancien et nous. L'écriture, la raison, les dieux, Paris : Hachette

IBRAHIM, A.H. (1990), Questions posées par l'arabe à une théorie générale des systèmes d'écriture, in Pour une théorie de la langue écrite, Paris : CNRS, 225-232.

- (1994), L'alphabétisation dans un contexte diglossique arabe, in Proceedings of the Workshop on Contexts of Literacy (Nice, 21-24 September 1994), 205-17.

JOUAD, H. (1987), «Les tribulations d'un lettré en pays chleuh », E.D.B., n 2, $27-41$.

MISKÉ, A.-B. (1970), Al Wasît. Tableau de la Mauritanie au début du XXè siècle, Paris : Klincksieck.

NAIT-ZERRAD, K. (1998), Lexique religieux berbère et néologie : un essai de traduction partielle du Coran, Milan : Centro Studi Camito-Semitici.

OULD CHEIKH, A.W. et SAISON, B., (1987), «Vie(s) et mort(s) de al-Imâm al-Hadrâmi. Autour de la postérité saharienne du mouvement almoravide (XI - XVII ${ }^{\mathrm{e}}$ siècle) », Arabica, XXXIV, 448-79.

OULD CHEIKH, A.W. (1985), Nomadisme, Islam et Pouvoir politique dans la société maure précoloniale $\left(\mathrm{XI}^{\mathrm{e}}\right.$-XIX ${ }^{\mathrm{e}}$ siècles). Essai sur quelques aspects du tribalisme, Thèse de sociologie, Paris $\mathrm{V}$.

RENFREW, C. (1994), La diversité linguistique, in Pour la science, 197 : 68-72.

SIRAN, J.-L. (1998), " C. r. de De la voix au texte. L'ethnologie contemporaine entre l'oral et l'écrit, éd. N. Belmont \& J.-Fr. Gossiaux, Paris, éd. du CTHS, 1997, 255 p., L'Homme, 147, 312-4.

TAINE-CHEIKH, C. (1994a), "Pouvoir de la poésie et poésie du pouvoir - le cas de la société maure », M.A.S. (G.E.L.L.A.S.), 6 (N. S.), 281-310.

- (1994b), «Les langues comme enjeux identitaires », Politique Africaine, 55 (Mauritanie : Un tournant démocratique ?), 57-65.

- (1995), Poésie et musique, in Notre Librairie. Littérature mauritanienne, Paris : C.L.E.F., 194-201.

- (1997), Pratiques de l'arabe et processus identitaires en Mauritanie, in Plurilinguisme et identité(s). Le cas du Maghreb, Mont-Saint-Aignan (2-3 mai 1996) : P.U.R., 23, 67-79.

- (1999), Le zénaga de Mauritanie à la lumière du berbère commun, in Afroasiatica Tergestina (Proceeding of the 9th Italian Meeting of Afroasiatic Linguistics held in Trieste, 23-24 Aprile 1998), M. Lambertini \& L. Tonelli, 299-324.

TAMARI, T. (1996), «L'exégèse coranique (tafsîr) en milieu manding », I.S.S.S., 10, 43-79. 